\title{
Effect of Risk-Predictive Haptic Guidance in One-Pedal Driving Mode
}

\author{
Y. Saito - P. Raksincharoensak
}

Received: date / Accepted: date

\begin{abstract}
The research presented in this article focuses on the design of a driver support system for risk-predictive driving under a potentially hazardous situation for a pedestrian who crosses a road from the driver's blind spots. Our aim is to develop a system that would cooperate with the driver in leading the normative speed calculated by the co-driver function. The design philosophy of haptic guidance is to communicate to the drivers the potentially hazardous situation through tactile cues from the active gas pedal and to assist drivers to in preparing for possible road surprises. We intended to combine the algorithm of the haptic feedback loop with the functionality of the one-pedal driving mode interface. Three design issues for the haptic guidance system can be distinguished: the design of a one-pedal driving mode based on a one-pedal operation; the modeling of risk-predictive driving behavior; and the haptic feedback algorithm with active gas pedal. We tested our system in human-in-the-loop experiments in a driving simulator to investigate (1) the effect of the onepedal driving mode interface on the driver behavior and (2) the effect of haptic guidance support on the driver behavior. From the results of our experiments, we confirmed that haptic guidance can improve the riskpredictive driving performance for a slowdown task via the one-pedal driving mode.
\end{abstract}

\section{Y. Saito}

Department of Mechanical Systems Engineering, Tokyo University of Agriculture and Technology, Tokyo 184-8588

Tel.: +81-42-388-7176

Fax: +81-42-388-7425

E-mail: y-saito@cc.tuat.ac.jp

P. Raksincharoensak

Department of Mechanical Systems Engineering, Tokyo University of Agriculture and Technology, Tokyo 184-8588
Keywords Automation · Human machine cooperation · Shared control · Haptic guidance · Force feedback · Potential risk

\section{Introduction}

Pedestrians are exposed to accident risks when crossing a road in urban areas, owing to the shared road spaces (Rankavat and Tiwari 2016). One of the most common types of traffic fatalities (NPA 2017) is being hit in a traffic accident while walking along the road (34.9\%). As the mortality rates rise sharply when the collision velocity for pedestrians with reduced impact resistance is greater than $30 \mathrm{~km} / \mathrm{h}$ (Centre 2006), it is important to anticipate the hidden contextual driving risks.

In time-critical situations, the existing autonomous emergency braking (AEB) systems implement a brake intervention at the last second on behalf of the driver to avoid crashing. However, AEB may not be able to satisfactorily realize the required functions owing to time constraints (i.e., delayed reaction times and time required for braking distance), when pedestrians cross a road from a point that falls in the blind spot of the assistance system. When expert drivers are confronted with uncertainty, they naturally seek to reduce the uncertainty by obtaining more information and attempting to fit their current driving context into a pre-existing category that they have already developed (McDermott 2010). Based on context information, e.g., parked vehicles in an urban area, they estimate the probability of possible road surprises, e.g., a playing child dashing out. If necessary, they take preventive measures that depend on the current driving context, such as decreasing the velocity. They ensure that "sufficient braking distance is available in case of an occurrence of one of the possible 


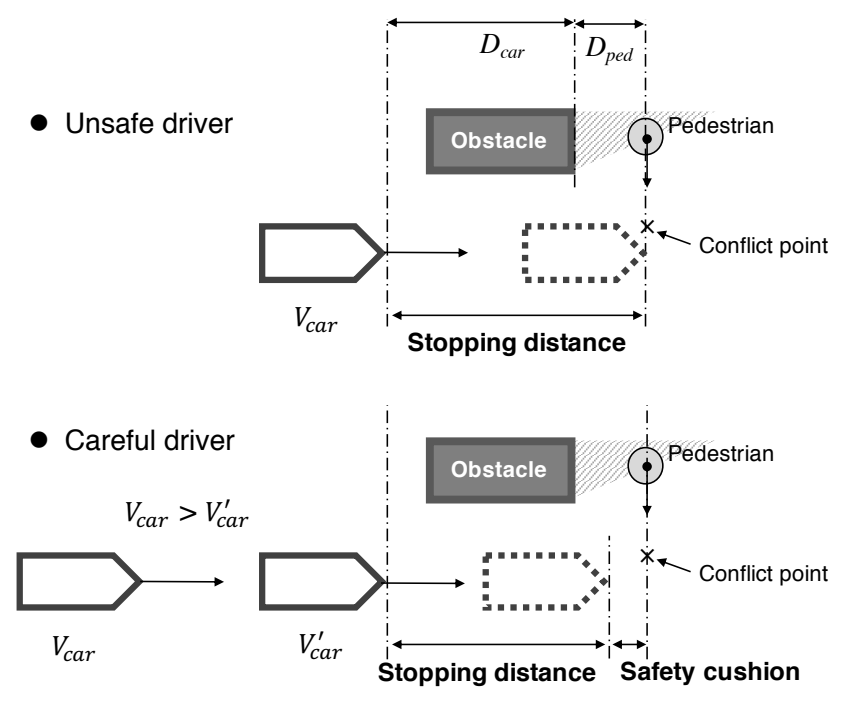

Fig. 1 Metaphorical safety cushion.

road surprises" (AAA-Foundation 2006; GmbH 2015). The additional braking distance that becomes available due to this preventive driver action is referred to as a "safety cushion" (Raksincharoensak and Inoue 2017), as shown in Figure 1. Our study goal is to develop an advanced driver assistance system (ADAS) to attain the risk-predictive driving based on the predictions of a situation's criticality.

In previous research, an active deceleration support (Saito and Raksincharoensak 2016a,b), based on codriver functions (Inoue et al. 2017), has been developed. We herein focused on assessing the potential risk for a pedestrian who crosses the road from a driver's blind spots in the absence of communication between the driver and pedestrian. When the ADAS determines that the vehicle is approaching a potentially hazardous situation while maintaining a velocity higher than the reference velocity calculated by defining the worst-case scenario, the ADAS implements deceleration control and assists the drivers in extending their capability for only the limited time of the hazardous situation, extending a time margin for reaction in case a potential yet unexpected critical situation occurs. Here, we mention a safety-first policy in that although it is clear that always assuming the worst-case scenario is effective for improving proactive safety, the concern is whether the choice of scenario is socially acceptable. That is, it is extremely rare for drivers to encounter a situation in which an "invisible" pedestrian crosses the road. Meanwhile, the damage resulting from the event is obviously unacceptable. In this context, the proposed active deceleration support concerns the following issues (Saito and Raksincharoensak 2016a):
- Although the ADAS implements the brake intervention with visual and auditory feedback (Ito et al. 2017) for only a limited time, the drivers may perceive most instances of such assistance as false positives. As drivers have various motives in operating the gas or brake pedals, the countermeasures to minimize potential risks may be perceived as annoying by the driver.

- Owing to partially automated control, human-outof-the-loop problems such as those arising from the loss or reduction in situation awareness, lack of vigilance (see, e.g., Sarter and Woods 1995; Endsley and Kiris 1995; Merat and Jamson 2009), over-reliance, and over-trust (see, e.g., Hollnagel and Woods 2005; Inagaki and Itoh 2013) must be addressed by an early intervention approach.

One type of human-machine interaction to always remain in the control loop, and to continuously interact with feedback via haptics is shared control (Abbink et al. 2012; Petermeijer et al. 2015); this concept has been extended to the framework of "shared and cooperative guidance and control" (Flemisch et al. 2014, 2016). In speed-control situations, an active gas pedal with a force feedback can be useful to establish the counterforce, which is dependent on the deviation relative to a normative behavior; thus, the feedback allows for the perception of force and force-slope changes (Mulder et al. 2011). The existing haptic gas pedal supports include the intelligent speed adaptation (ISA) that provides haptic information related to speed difference corresponding to a normative speed limit (Verwey et al. 1993; Adell et al. 2008; Vlassenroot et al. 2007), a car-following support that provides haptic information on the time-headway $(T H W)$ and time to contact $(T T C)$ relative to a leading vehicle (Mulder et al. 2010, 2011), and an eco-driving support that provides haptic feedback to guide a driver toward the idealized throttle angle (Jamson et al. 2013).

As a solution to the abovementioned issues, the paper is focused on designing a haptic guidance support system that assists in inducing the risk-predictive driving behavior and also reflects the drivers' intention by continuously interacting with the support system. The design philosophy of haptic guidance is to communicate to the drivers about potentially hazardous situations through tactile cues from the active gas pedal in maintaining the human-in-the-control loop for the slowing down task and to assist drivers to prepare for possible road surprises. This paper aims to demonstrate the effectiveness of the risk-predictive haptic guidance on a speed-control performance. This paper is organized as follows: In the following section, the design of the haptic guidance system with an active gas pedal is described in 


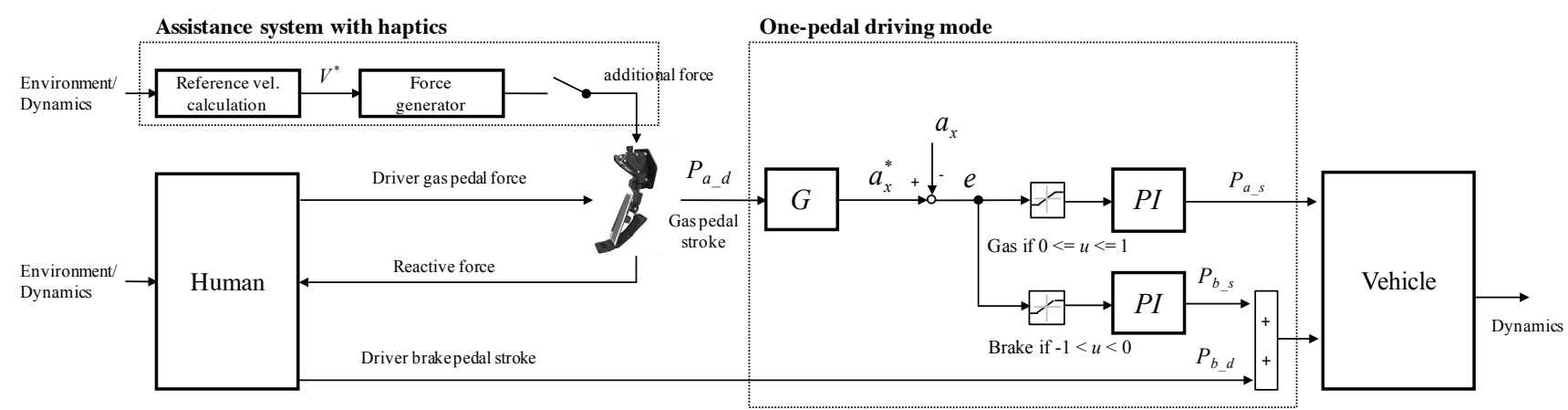

Fig. 2 Block diagram of the risk-predictive haptic guidance system.

detail; a one-pedal driving mode interface is proposed, and we intend to combine the algorithm of the haptic feedback loop with the functionality of the proposed one-pedal driving mode interface. In this context, we had two objectives:

- To investigate the effect of the one-pedal driving mode on the driver behavior.

- To investigate the effect of the haptic guidance support that provides the driver with continuous potential risk information with respect to the blind areas, on the driver behavior.

Two experiments are involved in the investigation of the two issues: Experiment 1, and Experiment 2. We tested our system in human-in-the-loop experiments in a sixmotion-base driving simulator. The two experimental results and discussions are presented herein, as well as our conclusion.

\section{Risk-Predictive Haptic Guidance}

Three important design issues can be highlighted for a risk-predictive haptic guidance support system:

1. Design of the one-pedal driving mode: based on the one-pedal operation.

2. Modeling of risk-predictive driving behavior: the codriver function generates a referenced terminal velocity in a given driving situation with blind areas.

3. Haptic feedback algorithm with active gas pedal: the interface converts from the potential risk information to force information.

\subsection{One-Pedal Driving Mode}

What is a smart driving behavior? As per Young (2011), a smart driving behavior involves the following: (a) planning ahead to avoid stopping, (b) using moderate engine speeds and a uniform throttle for a steady driving, (c) avoiding sharp braking, and (d) using engine braking for a smooth deceleration. The traditional twopedal system, with separate gas and brake pedals, is technically simple; however, this system also has certain disadvantages. The driver's action for a slowdown task can be approximately divided into two aspects: 1) use of friction braking, and 2) use of engine braking. When the driver uses the friction braking, he/she is required to perform a foot-switching operation from the gas pedal to the brake pedal; consequently, a reaction time occurs corresponding to the movement of the foot-switching operation. If the driver intends to mostly avoid this foot-switching operation, he/she needs to plan ahead; meanwhile, the driver requires a considerable amount of time to use engine braking to reach the targeted speed. Moreover, a potential risk of depressing on the wrong pedal exists. Nevertheless, some electronic vehicles have simplified the longitudinal driving task by affording the "one-pedal operation," which avoids the necessity to perform the brake-pedal action to slow down. One of the features of such electronic vehicles is regenerative braking with an energy recovery mechanism. When the driver releases the gas pedal, the vehicle immediately begins to slow down, similar to the application of friction braking. In this study, a one-pedal driving mode is applied to a normal gasoline-powered vehicle, with its interface represented as the block diagram indicated in Fig. 2. The interface is realized by the stroke (drive) by wire to generate the one-pedal operation.

1. One-pedal operation: The driver's gas pedal stroke is converted to the targeted acceleration through the transfer characteristic $G$, indicated in Fig. 2. Fig. 3 shows the relationship between the gas-pedal stroke position and the targeted acceleration/deceleration. The driver's gas pedal stroke can be classified over three ranges: i) stroke range designed for slowdown task, ii) stroke range designed for steady driving task, and iii) stroke range designed for accelera- 


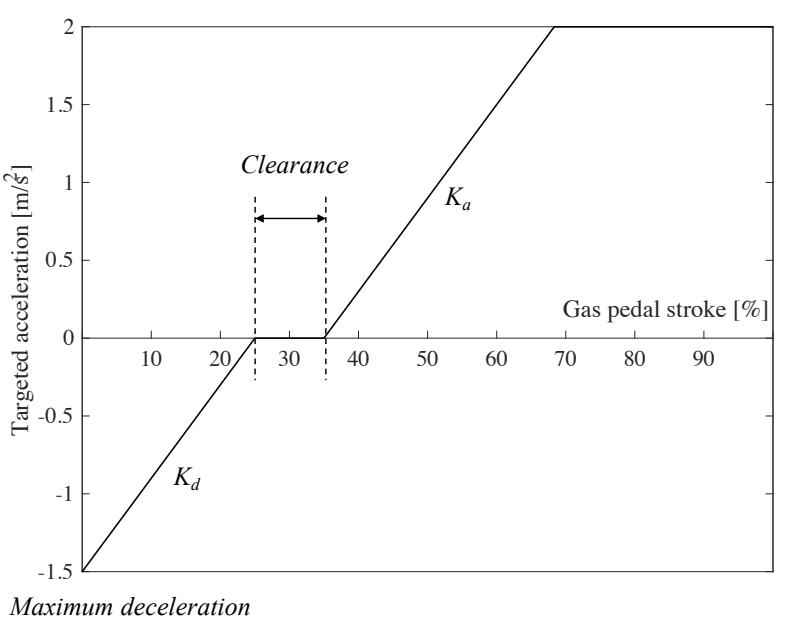

Fig. 3 Relationship between targeted acceleration and gas pedal stroke.

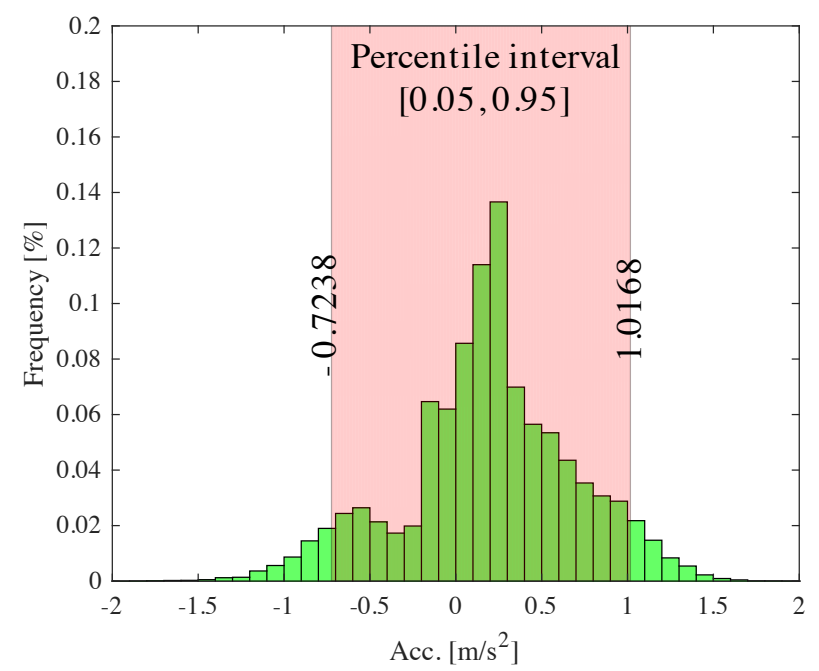

Fig. 4 Acceleration/deceleration. The naturalistic driving data on six driving instructors were collected at a Japanese urban area (Akagi and Raksincharoensak 2015). Participants drove a car equipped with a controller area network (CAN) data logger and several sensors on an urban road with a distance of $5.4 \mathrm{~km}$.

tion task. The parameters related to maneuverability are as follows: maximum deceleration, maximum acceleration, clearance range, gain $K_{d}$, and gain $K_{a}$. We assume the targeted acceleration/deceleration to take on values between -1.5 and $2 \mathrm{~m} / \mathrm{s}^{2}$; most of the acceleration/deceleration required in non-critical driving contexts cover the range, as indicated by Fig. 4. The stroke range designed for the steady driving task is the $10 \%$ range between the gas-pedal stroke range of $25 \%$ and $35 \%$ (as shown in Fig. 3),
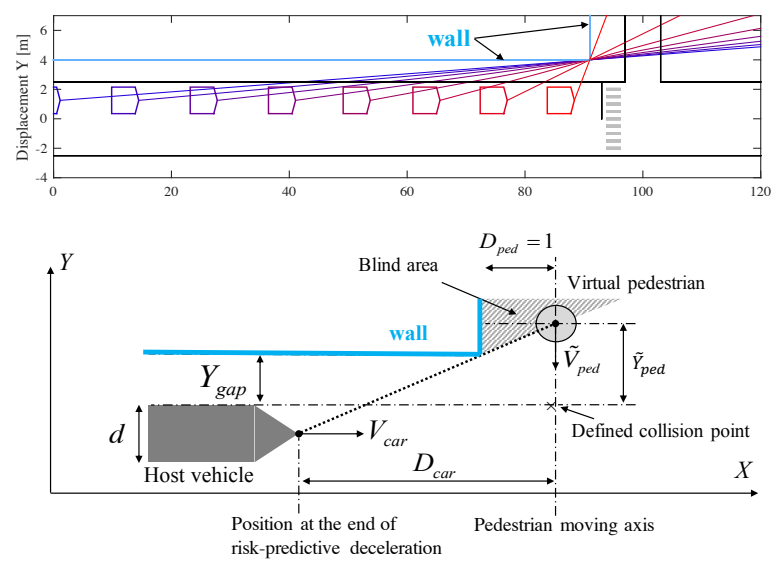

Fig. 5 Geometrical relationship between host vehicle, unsignalized intersection, wall, and blind area.

and the gains $K_{d}$ and $K_{a}$ are 0.06 . These values were designed through a preliminary experiment.

2. Stroke by wire: The error, e, between the determined and the actual acceleration/deceleration is converted to a virtual pedal stroke through a $P I$ controller. Based on the sign (positive or negative) of $e$, the virtual gas-pedal stroke, $P_{a_{-} s}$, or virtual brake-pedal stroke, $P_{b_{-} s}$, is input to the actuator of the vehicle. The strategy for combining the driver's input brake-pedal stroke, $P_{b_{-} d}$, and the machine's virtual brake-pedal stroke, $P_{b_{-} s}$, for the slowdown task can be expressed as follows:

$$
P_{b \_a c t}(t)=P_{b_{-} d}(t)+P_{b_{-} s}(t)
$$

\subsection{Modeling of Risk-Predictive Driving Behavior}

Based on previous research (Inoue et al. 2017), the modeling of risk-predictive driving behavior is briefly described. Fig. 5 illustrates the risk of collision with pedestrians who initiate a road crossing. As the vehicle approaches an intersection with no signals (an "unsignalized" intersection), the area with poor visibility reduces. We can set the collision point, indicated in Fig. 5, by assuming the worst-case scenario wherein a "darting-out" pedestrian collides with the left front corner of the vehicle. The lateral gap, $\tilde{Y}_{p e d}$, between the pedestrian and the collision point changes sequentially depending on the lateral gap, $Y_{\text {gap }}$, between the wall and the car, and the longitudinal gap, $D_{\text {car }}$. If a pedestrian crosses the road from a lateral position in which the driver can first perceive the darting-out of the pedestrian, a possible collision condition can be simply defined as follows:

$T_{t c \_p e d}(t)=T_{t c \_c a r}(t)$ 
where $T_{t c-p e d}(t)$ represents the time required for the pedestrian at his/her current speed to reach the collision point if the current traveling direction was maintained, and $T_{t c_{-} \text {car }}(t)$ represents the time required for the vehicle to reach zero $\mathrm{km} / \mathrm{h}\left(V_{\text {final }}\right)$ at the collision point under the assumed avoidance behavior (i.e., the reaction time, $\tau=0.6 \mathrm{~s}$ and maximum deceleration, $a_{\max }=-6 \mathrm{~m} / \mathrm{s}^{2}$ ). The following condition must be satisfied:

$V_{\text {car }}\left(t^{*}\right) \cdot \tau-\left(V_{\text {car }}^{2}\left(t^{*}\right)-V_{\text {final }}^{2}\right) / 2 a_{\max }=D_{\text {car }}\left(t^{*}\right)$

where $t^{*}$ represents the time when $T_{t c \_c a r}(t)$ becomes less than $T_{t c \_p e d}(t)$ for the first time. In other words, the terminal velocity, $V^{*}$, is determined as follows:

$V^{*}=V_{\text {car }}\left(t^{*}\right)=-a_{\max }\left(-\tau+\sqrt{\tau^{2}-2 D_{\text {car }}\left(t^{*}\right) / a_{\max }}\right)$

The referenced terminal velocity, $V^{*}$, is defined as the velocity at which a collision with the darting-out pedestrian is avoidable when the driver implements a braking action under the defined conditions. The terminal velocity, $V^{*}$, at the distance, $D_{\text {car }}\left(t^{*}\right)$, is dependent on the lateral gap, $Y_{\text {gap }}$, related to the degree of poor visibility, the pedestrian's walking speed, $\tilde{V}_{\text {ped }}$, and the evasive action parameters, $\tau$ and $a_{\max }$. Fig. 6 shows the plot of the referenced terminal velocity, $V^{*}$, with respect to the lateral gap, $Y_{\text {gap }}$; the dashed line denotes the velocity, $V^{*}$, when $\tilde{V}_{\text {ped }}=1.5 \mathrm{~m} / \mathrm{s}$. As we assumed stringent conditions, a lower terminal velocity is required. In this study, the $\tilde{V}_{\text {ped }}$ was set at $1.5 \mathrm{~m} / \mathrm{s}$ as the mean walking speed of a pedestrian based on the analysis of relevant near-crash events (Saito and Raksincharoensak 2016a). Thus, based on the calculation process described in (Inoue et al. 2017), the co-driver function generates a referenced terminal velocity in a given driving situation with blind areas.

\subsection{From Risk Information to Haptic Information}

The drivers are given information on the latent hazards through a haptic feedback loop with an active gas pedal. The potential risk can be simply expressed as a function of the current velocity, $V(t)$ :

$P R(t)= \begin{cases}V(t) / V^{*}-1 & \forall V(t)>V^{*} \\ 0 & \forall V(t) \leq V^{*}\end{cases}$

If $V(t)$ is less than $V^{*}$, a collision with a darting-out pedestrian is avoidable when the assistance system activates the conventional AEB. However, when $V(t)$ assumes a value larger than $V^{*}$, a collision with the pedestrian is unavoidable even under the assumption that the

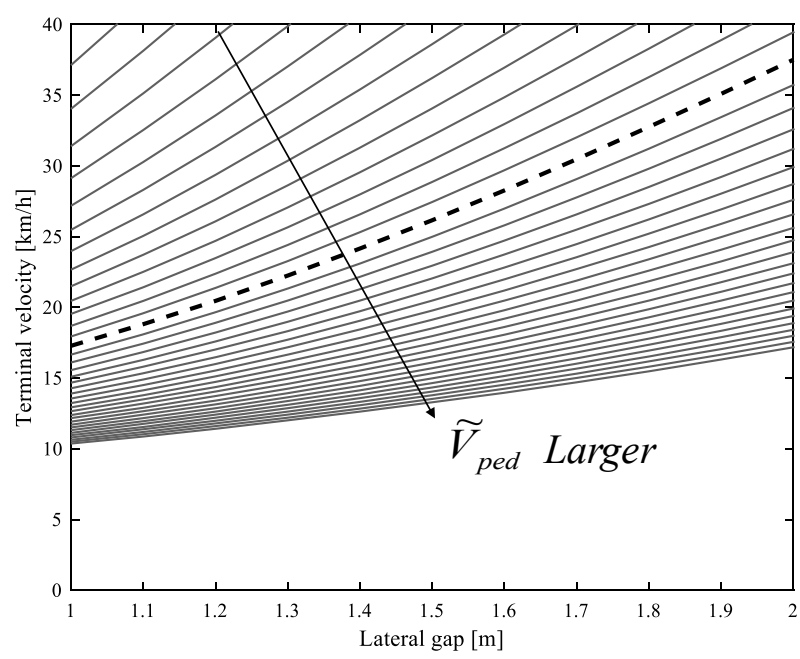

Fig. 6 Terminal desired velocity with respect to lateral gap.

AEB is activated; the collision velocity corresponds to the worst-case scenario.

The haptic feedback manner can be categorized into two types (Mulder et al. 2011, 2010): a) force feedback, and b) stiffness feedback. Mulder et al. (2011) have proposed an active deceleration support based on the force and stiffness feedback approaches, to provide drivers with continuous car-following information, which was transformed into the haptic information from the $T H W$ and TTC values. This paper proposes the haptic guidance support via the stiffness feedback. The additional force, $F_{\text {stiff }}$, based on the gas-pedal stiffness feedback, is given as follows:

$$
F_{\text {stiff }}(t)= \begin{cases}K_{\max } \cdot P_{a_{-}}(t) & \forall P R(t) \geq 1 \\ K_{\max } \cdot P R(t) \cdot P_{a_{-} d}(t) & \forall 0 \leq P R(t)<1\end{cases}
$$

The additional force is input to the active gas pedal through the transfer function of a primary-delay system. As can been inferred from Eqs. (5) and (6), the stiffness feedback algorithm does not generate any force when $P R(t)=0$. When the function $P R(t)$ assumes a value larger than zero, the additional force is increased depending on the driver's input stroke position, $P_{a_{-} d}(t)$. The degree of additional force is characterized by the maximum additional stiffness, $K_{\max }(=2)$, on the gas pedal. Let us suppose a moving vehicle is approaching an intersection with poor visibility. Subsequently, we set the lateral gap, $Y_{\text {gap }}$, between the left side of vehicle and the wall is observed as $1.5 \mathrm{~m}$, for which the referenced terminal velocity, $V^{*}$, calculated by the driver model is $26.3 \mathrm{~km} / \mathrm{h}$. In this case, the drivers can feel an additional force, $F_{\text {stiff }}$, depending on the $P R(t)$ 


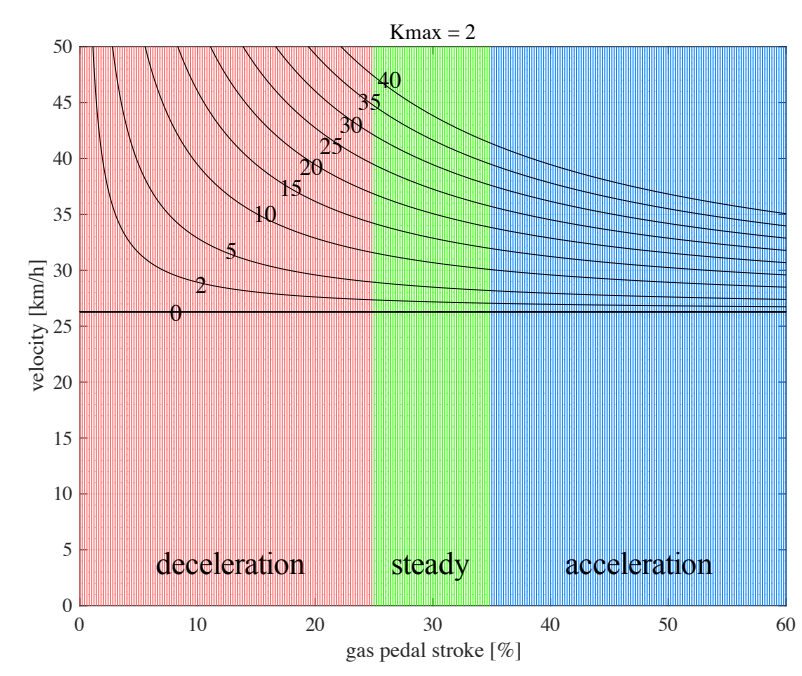

Fig. 7 Force lines (N) with respect to the current velocity $(\mathrm{km} / \mathrm{h})$ and the depression of the gas pedal (\%). The red area means the stroke range for deceleration, the green area means the stroke range for steady, and the blue area means the stroke range for acceleration.

and the driver's input stroke position, $P_{a_{-} d}(t)$. The relationship between the additional force, current velocity, and driver's input stroke position can be expressed as in Fig. 7. The haptic feedback algorithm is expected to induce the risk-predictive driving behavior by using the stroke range designed for a slowdown.

\section{Experiment 1: Car-following}

We collected the driver behavior data when using the one-pedal driving mode interface to assessing the usefulness of a single pedal task over a two-pedal task. The experiment was performed with the approval of the ethics committee at our university.

\subsection{Apparatus}

We used a six-motion-base driving simulator that consists of a host computer, a visual and an audio system, a steering system and a motion controller. The active gas pedal was installed in this simulator. The host computer calculated the vehicle dynamics based on the input of the driver operational interfaces and consequently generated the driving screen images. The field of the driving view was approximately $180^{\circ}$. In addition, any scene or traffic situation was reconstructed by setting the road environment and the traffic flow of other vehicles.

\subsection{Participants}

We recruited 14 healthy volunteer drivers between the ages of 22-40 who periodically drove (S1-S14, 13 males and 1 female, mean age: 25.5 years, s.d.: 5.24$)$.

\subsection{Design, Tasks, and Procedure}

A within-subjects design was used for this experiment; the independent measure was the driving condition: driving with a traditional two-pedal interface, and driving with the one-pedal interface. The participants were divided into two groups: (a) In the first group, the participants drove a car equipped with a traditional twopedal interface; subsequently, they drove a car equipped with the one-pedal interface. (b) In the second group, the participants first drove the car equipped with the one-pedal interface and then the car equipped with the traditional two-pedal interface. Regarding the conditions pertaining to the traditional two-pedal interface, the participants could use the friction braking with the brake pedal, and the engine braking with the foot off the gas pedal; the maximum deceleration with the foot off the gas pedal was approximately $-0.2 \mathrm{~m} / \mathrm{s}^{2}$. Regarding the conditions pertaining to the one-pedal interface, the participants could also use the brake pedal according to the circumstances because the maximum deceleration with the foot off the gas pedal was approximately -1.5 $\mathrm{m} / \mathrm{s}^{2}$; the participants were not prevented from using the brake pedal.

In the simulations, the participants drove a car equipped with an automatic transmission on a one-way road with two lanes in an urban area. On either side of the road, buildings, pedestrians, and signal intersections were present (Fig. 8). The primary task of the participant was to follow a lead car at his/her intended $T H W$. The lead car traveled with a predetermined speed profile between 0 and $50 \mathrm{~km} / \mathrm{h}$, and between -1 and $1.2 \mathrm{~m} / \mathrm{s}^{2}$, and the carfollowing scenario included a stop-and-go at the signal intersection. The participants were required to drive at a constant $T H W$ as far as possible. In addition, when the $T H W$ value exceeded 5 seconds, a translucent rectangle was displayed on the rear end of the lead car, which aided in maintaining the car-following condition. Thus, the participants were required to drive such that the translucent rectangle would not be displayed.

Informed consent was obtained from all participants. First, the participants underwent training trials for approximately $15 \mathrm{~min}$ to familiarize themselves with the simulator. Next, in group (a), the training trial of the car-following situation was conducted only once. After the training trial, two repetitions were recorded for the condition with the traditional two-pedal interface under 
the car-following situation (two-pedal_1, two-pedal_2). Subsequently, the participants attended a lecture on the one-pedal driving mode interface. After the lecture, the training trials to familiarize the participants with the one-pedal driving mode were performed for approximately $40 \mathrm{~min}$. The training trials included 1) driving freely with the one-pedal driving mode, 2) driving at the specified speed with the one-pedal driving mode (speed tracking task requiring feedback control), and 3) stopping at the specified stop line without using the brake pedal as far as possible (stopping task requiring feedforward control), and 4) driving at a carfollowing situation with the one-pedal interface; the training trial of the car-following situation was conducted only once. After these training trials, two repetitions were recorded for the condition with the one-pedal interface under the car-following situation (one-pedal_1, one-pedal_2). Group (b) followed the reverse procedure of that followed by group (a). Finally, the participants answered the NASA-TLX questionnaire (Hart and Staveland 1988) (Mental Demand, Physical Demand, Temporal Demand, Performance, Effort, and Frustration). The experiment required approximately $1.5 \mathrm{~h}$ per participant to complete.

\subsection{Hypotheses}

The car-following task comprised of two major phases: the following phase to maintain a designated gap between the vehicles, and the approach phase to approach a lead car. We hypothesized that if the one-pedal driving mode improved the driving performance, particularly in the approach phase, i.e., corresponding to a small value of $T T C$, this would be because the participant's reaction time against the deceleration of the lead car improved over that with the traditional twopedal interface. In the following phase, if a significant difference in $T H W$ occurred between the two conditions, this would be because the participants adopted a suitable compensation behavior. If the participants have set aside a larger $T H W$ for driving with the onepedal interface when compared with that for the traditional two-pedal interface, this would be because they intended to perform the car-following tasks within the deceleration range $\in[-1.5,0]$ in order to avoid performing the brake-pedal action to slow down. If the participants maintained a smaller $T H W$ for driving with the one-pedal interface, this would be the result of a negative effect (Cacciabue and Saad 2008; OECD 1990) on the increased safety margin because of the increase in the deceleration range available with the gas pedal.

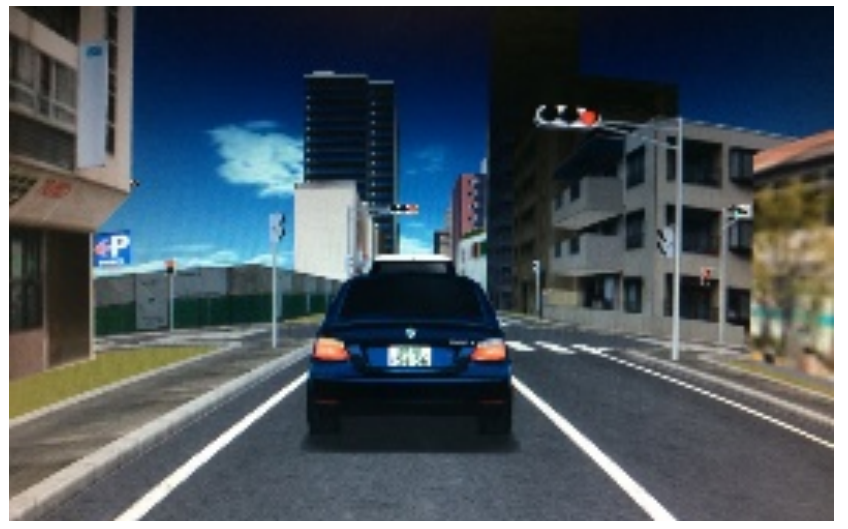

Fig. 8 Car-following scenario.

\subsection{Dependent Measures}

From the data recorded during the simulator experiment, we extracted a number of variables such as performance, reaction time, and control effort and determined the following:

- Mean value and standard deviation of $T H W$.

- Maximum value of inverse of TTC: This was calculated using the recorded data of the specified scenario when the lead car decelerated at $-1 \mathrm{~m} / \mathrm{s}^{2}$ to 0 $\mathrm{km} / \mathrm{h}$ from $40 \mathrm{~km} / \mathrm{h}$.

- Reaction time: The reaction time was defined as the time spent by the driver of the following car to exceed $-0.5 \mathrm{~m} / \mathrm{s}^{2}$ from slowing down of the lead car. It was calculated using the recorded data of the specified scenario when the lead car decelerated at -1 $\mathrm{m} / \mathrm{s}^{2}$ to $0 \mathrm{~km} / \mathrm{h}$ from $40 \mathrm{~km} / \mathrm{h}$.

- Foot-off gas pedal time: This parameter was calculated as a percentage of the total driving time.

- Actuating time of the brake pedal: Again, this parameter was calculated as a percentage of the total driving time.

These data were analyzed using a paired t-test for the driving condition (two-pedal_2, and one-pedal_2). The results with $p<.05$ are reported as significant.

\section{Experiment 1: Results and Discussion}

Figs. 9 (e) and (f) show the box plots for the mean and standard deviation, respectively, of the driver's intended $T H W$ between vehicles. The results of the paired $t$-test with the experimental condition applied to both the mean and standard deviation of $T H W$ show that the $T H W$ was not influenced by the traditional twopedal interface or the one-pedal driving mode interface. Fig. 9 (a) shows the box plot for the maximum value of 

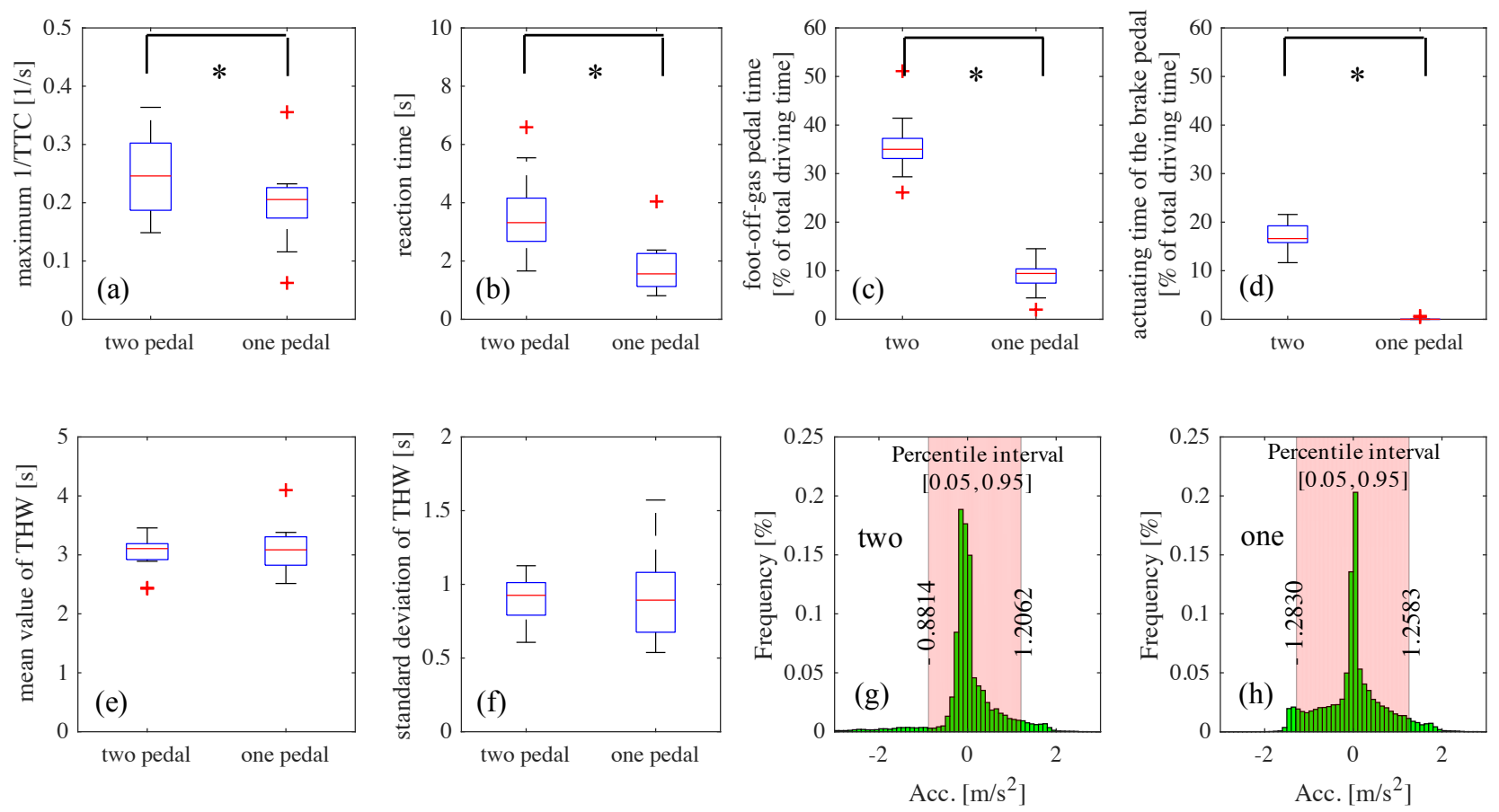

Fig. 9 Boxplot for maximum $1 / T T C$, reaction time, mean value of $T H W$, standard deviation $T H W$, foot-off-gas pedal time, actuating time of brake pedal, and frequency of acceleration. The frequency distribution of acceleration was depicted by using the data recorded from two repetitions for each condition.

the inverse of $T T C$. The results of the paired $t$-test with the experimental condition applied to the maximum $1 /$ TTC show significant differences $(t(13)=2.7613, p$ $<.05)$. The one-pedal driving mode interface has a significant effect on the maximum $1 / T T C$. As shown in Fig. 9 (a), the condition of the one-pedal driving mode interface yielded a lower maximum $1 / T T C$ when compared with that of the traditional two-pedal interface. Fig. 9 (b), which illustrates the box plot for the reaction time, also indicates significant differences $(t(13)=$ $5.2395, p<.05)$. Similar to the trends for the maximum $1 / T T C$, the condition of the one-pedal driving mode interface yielded a lower reaction time than the traditional two-pedal interface. Figs. 9 (c) and (d) show the box plots for the foot-off gas-pedal time and the actuating time of the brake pedal, respectively. A paired $t$-test with the experimental condition applied to both variables indicated significant differences in the foot-off gas pedal time $(t(13)=17.753, p<.05)$, and in the actuating time of the brake pedal $(t(13)=22.689, p<.05)$. Figs. $9(\mathrm{~g})$ and $(\mathrm{h})$ show the normalized frequency for the acceleration-and-deceleration process, for the twoand one-pedal cases, respectively. It was clear that the participants could effectively use the range between 1.5 and $0 \mathrm{~m} / \mathrm{s}^{2}$ when using the one-pedal driving mode interface. Fig. 10 shows the NASA-TLX ratings and

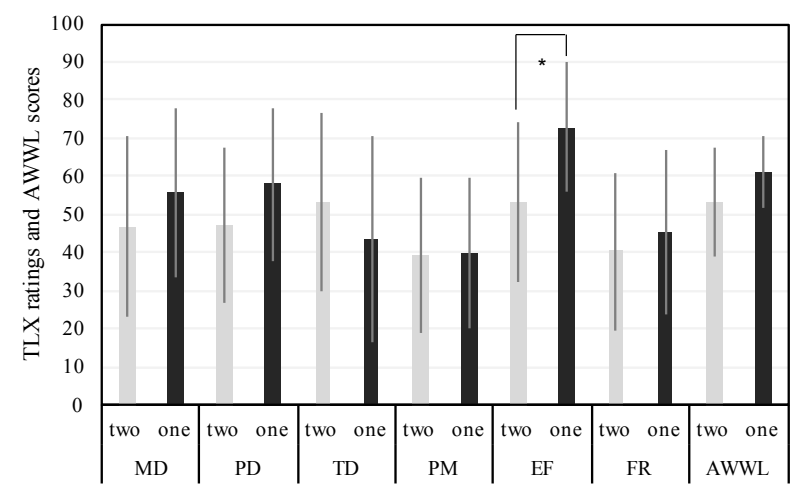

Fig. 10 NASA-TLX ratings and AWWL scores.

the adaptive weighted workload (AWWL) scores. The results of the paired $t$-test with the experimental condition applied to each variable (mental demand, physical demand, temporal demand, performance, effort, and frustration, and AWWL) indicated that the significant differences were found in the Effort $(t(13)=-3.3458, p$ $<.05)$.

The one-pedal driving mode interface was designed to simplify the longitudinal driving task by affording a one-pedal operation. The interface was realized by the stroke by wire to realize a one-pedal operation, and the driver's gas-pedal stroke was transformed to achieve the 
targeted acceleration. Three stroke ranges correspond to the acceleration, steady driving, and slowdown tasks. Here, we remark that the car-following task is primarily a combination of the following task and the approach task. From our statistical analysis of the standard deviation of $T H W$, it follows that car-following performance did not improve; no changes occurred in the standard deviation of the $T H W$ during the following task. However, from the statistical analysis of the maximum value of the inverse of $T T C$, it follows that the performance in the approach task improved; it is clear that the shorter reaction time contributed to the better performance when compared with that obtained with the traditional two-pedal interface because the participants could execute the slowing down task without performing the foot-switching operation from the gas pedal to the brake pedal. Therefore, the first hypothesis was validated: the one-pedal driving mode interface improved the driving performance in the approach task owing to reduction in the participant's reaction time against the deceleration of the lead car.

The second hypothesis was that if a significant difference in $T H W$ occurred between the two conditions, it would be because the participants adopted a compensation behavior. In our experiment, no change was observed in the THW during the following task; however, further experiments will be necessary in this regard. When driving a car with a one-pedal operation, a driver needs to train his/her mind to not move his/her foot to find the brake pedal. Thus, the training trials to familiarize the driver with the one-pedal driving mode interface were performed for approximately $40 \mathrm{~min}$, i.e., including the free-driving task, speed-tracking task requiring feedback control, and stopping task requiring feedforward control. When driving a car with the traditional two-pedal interface, the driver uses the engine braking by releasing the gas pedal even when he/she is maintaining a gap between his/her vehicle and the vehicle ahead. In other words, the drivers frequently repeat the operation of depressing and releasing the gas pedal. Meanwhile, when driving a car with the one-pedal operation, the drivers are required to keep depressing the gas pedal to maintain steady speed because the expansion of the deceleration region can be actuated only by the gas pedal. Thus, the driver's behaviors in the car-following task differ between the traditional twopedal interface and the proposed one-pedal interface. As observed from the statistical analysis of the footoff gas pedal time and the actuating time of the brake pedal, when using the one-pedal interface, it is clear that the participants could react by just operating the gas pedal most of the time during the following task; however, the foot-off gas pedal time [\% of the total driv- ing time], which involved a complete release of the gas pedal, was reduced when compared with that for the traditional two-pedal interface. As observed from the NASA-TLX ratings, the subjective control effort in the car-following task increased with the one-pedal driving mode interface; many participants commented that it was difficult for them to perform fine adjustments on the gas pedal and to train their mind to not move their foot to find the brake pedal. Therefore, although no changes occurred in the $T H W$ during the following as per our short-term analysis, long-term analyses in the real world or simulator environment are still lacking.

\section{Experiment 2: Risk-Predictive Driving}

We collected the driver behavior data when adding haptic guidance under the potentially hazardous situation, to analyze the effect/benefit of the risk-predictive haptic guidance. The experiment was performed with the approval of the ethics committee at our university.

\subsection{Apparatus}

As with the environment constructed in experiment 1 , the six-motion-base driving simulator was used in this experiment.

\subsection{Participants}

We recruited 18 healthy volunteer drivers between the ages of 22-40 who periodically drove (D1-D18, 17 males and 1 female, mean age: 26.2 years, s.d.: 5.42 ). Of the 18 people, 12 participants were drivers who participated in experiment 1 .

\subsection{Design, Tasks, and Procedure}

A within-subjects design was used for this experiment; the independent measure was the driving condition: driving with a traditional two-pedal interface without haptic guidance, and driving with the one-pedal interface with haptic guidance.

In the simulation, the participants drove on a oneway road with two lanes in an urban area. There were buildings, walls, and pedestrians on the road along with three intersections with no signals. The lateral gap between the wall and the center of the driving lane was 2.4 $\mathrm{m}$. The referenced terminal velocity when passing the intersection was set at $26.3 \mathrm{~km} / \mathrm{h}$. This was a scenario in which an oncoming vehicle occasionally approached 
the driver's vehicle. In this simulations, there was no event in which a pedestrian a suddenly crosses the road at each intersection. The participants were instructed to accelerate up to approximately $40 \mathrm{~km} / \mathrm{h}$, and they were required to pass the intersection while slowing and steering according to their own judgment.

Informed consent was obtained from all participants. First, the training trials to familiarize the participants with the simulator were performed for approximately 15 min. Subsequently, the participants drove the car equipped with the traditional two-pedal interface. Two repetitions were recorded for the driving with the traditional two-pedal interface without haptic guidance (without haptic guidance 1, and without haptic guidance 2). Next, the participants attended a lecture on the haptic guidance support with the one-pedal driving mode. After the lecture, the training trials to familiarize the participants with the one-pedal driving mode were performed for approximately $40 \mathrm{~min}$. The training trials included 1) driving freely with the one-pedal driving mode, 2) driving at the specified speed with the one-pedal driving mode (speed tracking task), and 3) following the lead car while maintaining a given distance with the one-pedal driving mode (car-following task). After the training trial with the guidance support was conducted once, two repetitions were recorded for the driving with the one-pedal interface with the haptic guidance (with haptic guidance 1, and with haptic guidance 2). Finally, the participants answered the acceptance questionnaire developed by J. D. van der Laan et al. (1997). The acceptability scale was a five-point rating scale. Participants indicated their opinions about the assistance system on a total of nine items (i.e., useful, good, effective, assisting, raising alertness, pleasant, nice, likable, and desirable). The scores for two acceptance dimensions, usefulness and satisfaction, were calculated. The experiment required approximately $1.5 \mathrm{~h}$ per participant to complete.

\subsection{Applied Haptic Guidance}

When the ADAS with a lean digital map (Ito et al. 2015) detects an intersection ahead including blind areas with the relative distance to the intersection being less than $70 \mathrm{~m}$, it provides an additional force to the active gas pedal; thus, the ADAS provided only the haptic feedback to guide the drivers toward the normative behavior.

\subsection{Hypothesis}

We hypothesized that if the participants could be led to the referenced terminal velocity calculated by the co-driver function through haptic guidance, this would be because they perceived the additional force, and recognized the additional force reduction (force-slope changes) as the current velocity approached closer to the referenced velocity.

\subsection{Dependent Measures}

From the data recorded during the simulator experiment, a number of variables were extracted:

- Minimum velocity, $V_{\min }$ : The minimum velocity was calculated using the recorded data while passing the unsignalized intersection including the blind areas.

- Collision velocity, $V_{c o l}$ : The haptic guidance support was tested only for situations in which the pedestrian did not cross the road because of the risk, in that it could create trauma for the participating drivers. In this study, assuming that the "virtual" pedestrian initiates a road crossing, the collision velocity, $V_{c o l}$, for the virtual pedestrian was calculated. We assume that the virtual pedestrian crosses the road at every sampling interval. The virtual pedestrian's velocity was set at $1.5 \mathrm{~m} / \mathrm{s}$. We assumed that the assistance system would implement the AEB (i.e., $\tau=0.6 s$ and $a_{\max }=-6 \mathrm{~m} / \mathrm{s}^{2}$ ) to avoid a crash when it determined that there was a possibility of collision with the pedestrian.

These data were analyzed using a paired t-test for the last intersection entry scenario in each condition (without haptic guidance 2 , and with haptic guidance 2 ). The results with $p<.05$ are reported as significant.

\section{Experiment 2: Results and Discussion}

Fig. 11 shows a typical example of the recorded data (participant D4) with haptic guidance using the active gas pedal. The plots show the lateral position, velocity [red: desired terminal velocity while passing the intersection, blue: actual velocity], acceleration, gas pedal stroke, brake pedal stroke, and additional force. The collision velocity is indicated by the colored bar. Gray indicates no possibility of collision; green indicates that the collision is avoidable under the assumption that AEB is activated; blue indicates that the collision velocity is less than $30 \mathrm{~km} / \mathrm{h}$; red indicates that the collision velocity is greater than $30 \mathrm{~km} / \mathrm{h}$. From Fig. 11, we can confirm that the participants could be led to the 

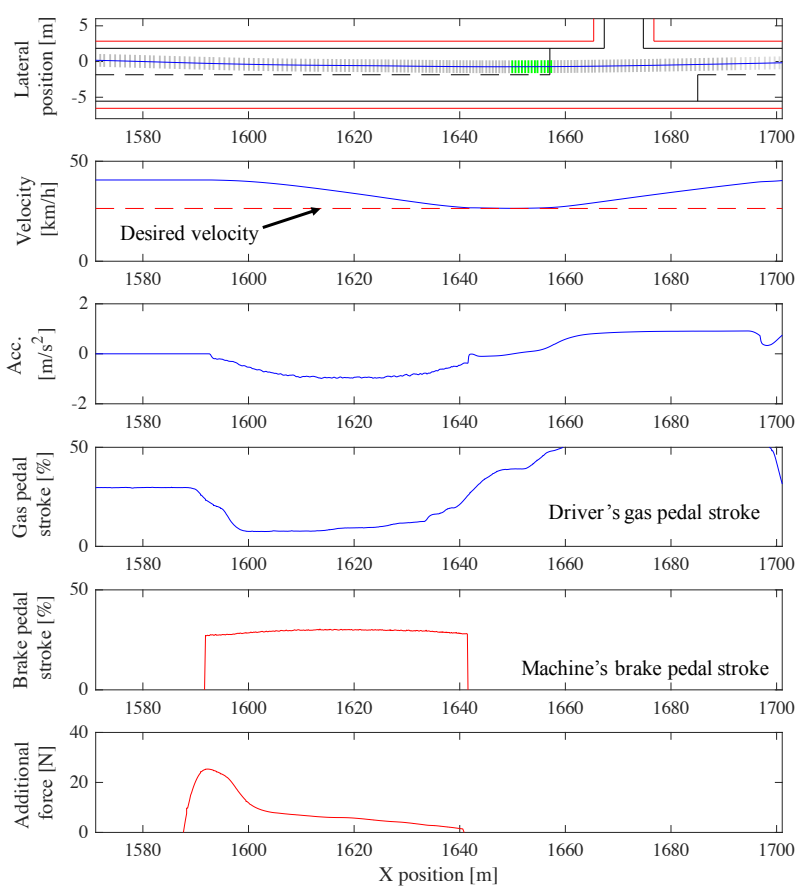

Fig. 11 Time-series data of D4 with haptic guidance.

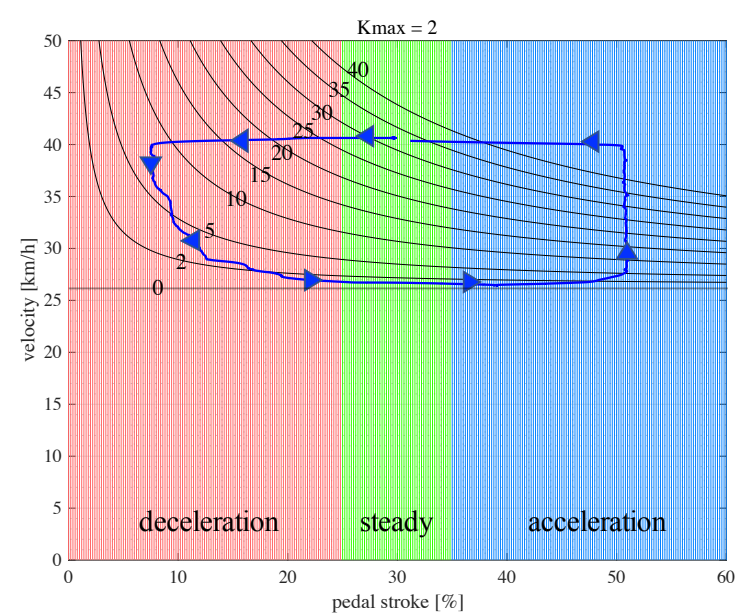

Fig. 12 Relationship between the pedal stroke position and the force lines $(\mathrm{N})$ with respect to the current velocity $(\mathrm{km} / \mathrm{h})$ and the depression of the gas pedal (\%).

referenced velocity by applying the additional force to the gas pedal; the ADAS assisted the drivers in obtaining the referenced velocity by using the stroke range designed for the slowdown task through the one-pedal driving mode. Thus, the vehicle slowed down without requiring the driver's foot-switching operation from the gas pedal to the brake pedal. Fig. 12 illustrates the relationship between the participant's pedal stroke position and the force lines $(\mathrm{N})$ with respect to the current velocity and the depression of the gas pedal (partici-
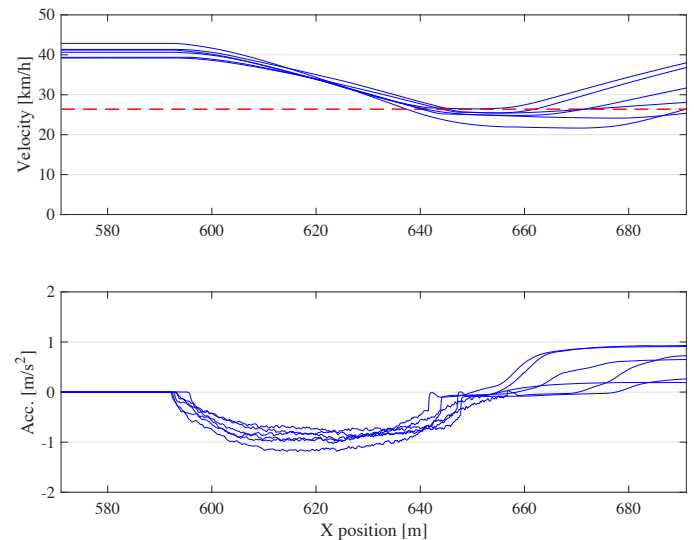

Fig. 13 All recorded trajectory data while passing the intersection (D4).
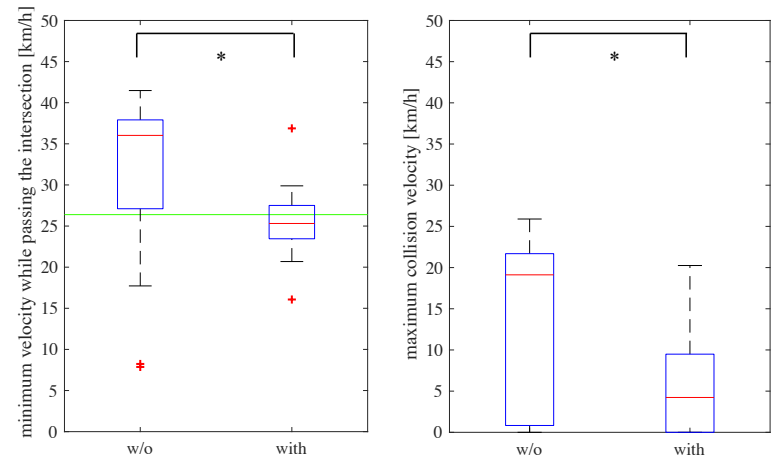

Fig. 14 Effect of minimum velocity and collision velocity.

pant D4), and the arrows denote the changes in the gas-pedal stroke position. Fig. 13 shows the trajectory data, i.e., velocity and acceleration, while passing the intersection. From Figs. 12 and 13, it appears that the haptic guidance support assisted the participant to perform a smooth deceleration. As a result of slowing down to the referenced terminal velocity, collision with the virtual pedestrian was avoidable under the assumption that AEB was activated. Fig. 14 shows the effects of the minimum velocity and collision velocity on the driving performance when passing the intersection adding or subtracting the haptic guidance support system. The paired $t$-test with the experimental condition applied to both variables showed significant differences in the minimum velocity $(t(17)=2.9262, p<.05)$, and in the collision velocity $(t(17)=2.2041, p<.05)$. In the minimumvelocity plot in Fig. 14, the green line denotes the referenced terminal velocity calculated by the driver model. It is clear that the participant drivers could be led to the referenced terminal velocity by the application of the additional force on the gas pedal. Finally, the re- 


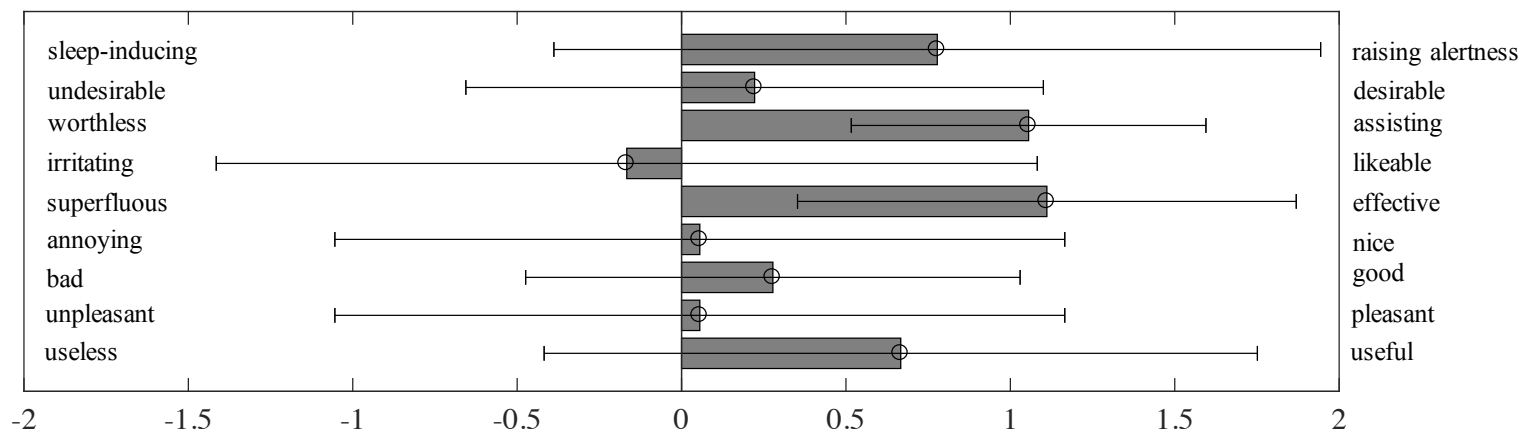

Fig. 15 Acceptance scores.

sults of the rating scores on the driver acceptance are shown in Fig. 15.

Haptic guidance was designed to guide a driver to adopt risk-predictive driving such that he/she could increase his/her safety margins. The support system afforded haptic information on the latent hazard through the haptic feedback loop with the proposed one-pedal driving mode interface, and the counterforce was dependent on the deviation relative to the referenced velocity. The hypothesis was that if the participants could be led to the referenced terminal velocity, this would be because he/she perceived the additional force and recognized the additional force reduction as the current velocity approached closer to the referenced velocity. From our statistical analysis of the minimum velocity while passing the intersection, we found that the participants could be led to the referenced terminal velocity through haptic guidance. Thus, our hypothesis was validated; the results indicate that drivers begin to slow down via the haptic feedback loop with gas-pedal stiffness feedback.

In the past decade, many studies have focused on assessing overt and/or potential risks in car-following and/or collision-avoidance situations (Mulder et al. 2010 , 2011; Takae et al. 2009). In contrast, our study focuses on assessing the potential risks in relation to hazards that cannot be observed by the driver or detected by the conventional AEB system. As mentioned in the introduction, the key aspect of our study is as follows: the possibility that the unrecoverable event for a driver will happen is extremely low, but the damage resulting upon the occurrence of the event is unacceptable. Therefore, the drivers may perceive most of these assistance inputs as false positives. The proposed assistance system only provides haptic feedback, without enhancing the situation awareness with auditory alerts and visual information, to avoid or reduce driver annoyance. Moreover, although the haptic feedback loop enables the driver to slow down, the driver determines the de- celeration for the slowdown task by adjusting the gaspedal stroke position via the one-pedal driving mode interface. As can be observed from our statistical analysis of the minimum velocity, the drivers could override the slowing down process initiated by the haptic feedback by depressing the gas pedal and/or releasing the gas pedal. The primary advantage of the proposed haptic guidance approach is that it assists in inducing risk-predictive driving behaviors and also reflects the intent of the driver by continuously interacting with the assistance system. As a solution to the issues to be addressed in the active deceleration support (Saito and Raksincharoensak 2016a), this proposed haptic guidance can be considered in the following manner: The important features of the system are that the active gas-pedal stroke is classified into three stroke ranges (acceleration, steady, and deceleration), and the haptic feedback and the one-pedal driving mode interface operate in unison to maintain the human-in-the-control loop for the slowing down task. The proposed method can also be applied to electric vehicles with one-pedal operation by merely mounting an active gas pedal to generate an additional force.

\section{Limitation and Future Works}

Our human-in-the-loop experiments had two objectives. In the car-following experiment, we investigated the effect of the one-pedal driving mode interface on the driver behavior. While we analyzed the short-term effects on the driver behavior, a long-term-effect analysis is still lacking. The behavioral adaptation needs to be investigated through long-term experiments. Moreover, many participants commented that it was difficult for them to maintain a steady driving owing to the "narrowness" of the stroke range designed for steady driving. Thus, it is necessary to improve the design of the 
one-pedal driving mode interface to enable good maneuverability.

In our risk-predictive driving experiments, we investigated the effect of the haptic guidance support on the driver behavior. The driving trial was repeated several times for each participant, and the targeted velocity while passing the intersection was set at $26.3 \mathrm{~km} / \mathrm{h}$. Consequently, it is necessary to investigate the effect of the guidance system on the driver behavior for different targeted velocities and/or different strengths of the haptic feedback. Thus, evaluations related to individual differences should be conducted, when a mismatch between the degrees of hazard anticipated by humans and by machines occurs owing to decision errors such as false assumptions and aggressive behaviors. The haptic guidance support was tested only for situations in which the pedestrian did not cross the road because this could otherwise be traumatic to the participating drivers. Each participant first practiced for the intersection entry scenario; subsequently, possible expected scenarios were also repeated several times. Therefore, it is necessary to evaluate the driver behavior in the case where an unexpected situation occurs. In addition to the experimental conditions, our assistance system was activated when it detected an intersection including the blind areas ahead with the relative distance to the intersection being less than $70 \mathrm{~m}$. The initiating condition should be changed according to the driving context. A long-term analysis of the driver acceptance and trust in the real world is also lacking. Finally, we recognized that the proposed assistance system can be useful for a wide range of age groups. Research covering a wide range of age groups is still lacking. In this regard, we have currently developed an experimental vehicle equipped with a number of environment sensors. We plan to address the issues related to the limitations above through field operations and testing.

\section{Conclusion}

Previous research (Saito and Raksincharoensak 2016a) has proposed active deceleration support as part of the risk-predictive braking maneuvers to reduce the potential risk of a pedestrian who crosses the road from the driver's blind areas in the absence of a communication device; the ADAS activates an early brake intervention to ensure a sufficient time margin for the driver to react in the case that an unexpected time-critical situation occurs. However, drivers may perceive most of these assistances as false positives due to the high uncertainty in the judgment of criticality. The primary contribution of our study is to present a haptic guidance design for risk-predictive driving, i.e., a slowing down task that enables drivers to increase their safety margins in a given situation. We intended to combine the algorithm of the haptic feedback loop with the functionality of the one-pedal driving mode interface. We tested our system in human-in-the-loop experiments in a driving simulator to investigate (1) the effect of the one-pedal driving mode interface on the driver behavior and (2) the effect of haptic guidance support on the driver behavior. From the results of our experiments, our conclusions are as follows:

- The one-pedal driving mode interface can be useful for simplifying the longitudinal driving task by affording the one-pedal operation, and it can improve the driver performance in the approach task in carfollowing.

- Haptic guidance with the active gas pedal assists in inducing risk-predictive driving behaviors and also reflects the driver's intention by continuously interacting with the support system.

Our risk-predictive haptic guidance can be valuable in coping with driving uncertainty and complexity in urban areas, although further research on the approach is required.

Acknowledgements This study has been conducted as a part of the research project "Autonomous Driving Intelligence System to Enhance Safe and Secured Traffic Society for Elderly Drivers" granted by Japan Science and Technology Agency. The authors would like to thank the agency for providing financial support.

\section{References}

AAA-Foundation. 2006. How risky is it? an assessment of the relative risk of engaging in potentially unsafe driving behaviors. Technical report.

Abbink, David A., Mark Mulder, and Erwin R. Boer. 2012. Haptic shared control: smoothly shifting control authority? Cognition, Technology $\& 3$ Work 14:1928.

Adell, Emeli, András Várhelyi, and Magnus Hjälmdahl. 2008. Auditory and haptic systems for in-car speed management - a comparative real life study. Transportation Research Part F: Traffic Psychology and Behaviour 11:445-458.

Akagi, Yasuhiro, and Pongsathorn Raksincharoensak. 2015. Stochastic driver speed control behavior modeling in urban intersections using risk potential-based motion planning framework. In 2015 IEEE Intelligent Vehicles Symposium (IV), 368-373.

Cacciabue, Pietro Carlo, and Farida Saad. 2008. Behavioural adaptations to driver support systems: a 
modelling and road safety perspective. Cognition, Technology \& Work 10:31-39.

Centre, OECD/ECMT Transport Research. 2006. Speed management report. Technical report, Paris.

Endsley, Mica R., and Esin O. Kiris. 1995. The out-ofthe-loop performance problem and level of control in automation. Human Factors 37:381-394.

Flemisch, Frank Ole, David A. Abbink, Makoto Itoh, Marie-Pierre Pacaux-Lemoine, and Gina Weßel. 2016. Shared control is the sharp end of cooperation: Towards a common framework of joint action, shared control and human machine cooperation. IFAC-PapersOnLine 49:72-77.

Flemisch, Frank Ole, Klaus Bengler, Heiner Bubb, Hermann Winner, and Ralph Bruder. 2014. Towards cooperative guidance and control of highly automated vehicles: H-mode and conduct-by-wire. Ergonomics $57: 343-360$.

GmbH, DEKRA Automobil. 2015. Strategies for preventing accidents on european roads. Technical report, ROAD SAFETY REPORT 2015 A future based on experience.

Hart, Sandra G., and Lowell E. Staveland. 1988. Development of nasa-tlx (task load index): Results of empirical and theoretical research. Advances in Psychology 52:139-183.

Hollnagel, Erik, and David D. Woods. 2005. Joint cognitive systems : foundations of cognitive systems engineering. Boca Raton, FL : Taylor \& Francis.

Inagaki, Toshiyuki, and Makoto Itoh. 2013. Human's overtrust in and overreliance on advanced driver assistance systems: A theoretical framework. International Journal of Vehicular Technology 951762:8 pages.

Inoue, Hideo, Pongsathorn Raksincharoensak, and Shintaro Inoue. 2017. Intelligent driving system for safer automobiles. Journal of Information Processing 25:32-43.

Ito, Takuma, Masahiro Mio, Kyoichi Tohriyama, and Minoru Kamata. 2015. Novel map platform based on primitive elements of traffic environments for automated driving technologies. Proc. the FASTzero'15: 3rd International Symposium on Future Active Safety Technology Toward zero traffic accidents, 2015 361:361-368.

Ito, Takuma, Tatsuya Shino, and Minoru Kamata. 2017. Information sharing to improve understanding of proactive braking intervention for elderly drivers. International Journal of Intelligent Transportation Systems Research.

Jamson, Hamish, Daryl L. Hibberd, and Natasha Merat. 2013. The design of haptic gas pedal feedback to support eco-driving. In Seventh International
Driving Symposium on Human Factors in Driver Assessment, Training, and Vehicle Design, 264-270.

Laan, Jinke D. Van Der, Adriaan Heino, and Dick De Waard. 1997. A simple procedure for the assessment of acceptance of advanced transport telematics. Transportation Research Part C: Emerging Technologies 5:1-10.

McDermott, Rose. 2010. Decision making under uncertainty. In Proceedings of a Workshop on Deterring Cyberattacks: Informing Strategies and Developing Options for U.S. Policy. The National Academies Press.

Merat, Natasha, and A. Hamish Jamson. 2009. How do drivers behave in a highly automated car? In International Driving Symposium on Human Factors in Driver Assessment, Training, and Vehicle Designcle Design, volume 5, 514-521.

Mulder, Mark, David A. Abbink, Marinus M. van Paassen, and Max Mulder. 2011. Design of a haptic gas pedal for active car-following support. IEEE Transactions on Intelligent Transportation Systems 12:268-279.

Mulder, Mark, Jasper J. A. Pauwelussen, Marinus M. van Paassen, Max Mulder, and David A. Abbink. 2010. Active deceleration support in car following. IEEE Transactions on Systems, Man, and Cybernetics - Part A: Systems and Humans 40:1271-1284.

NPA. 2017. Traffic accidents situation in 2016. Technical report, National Police Agency of JAPAN.

OECD. 1990. Behavioural adaptations to changes in the road transport system. Organization for Economic Co-operation and Development, Paris.

Petermeijer, Sebastiaan M., David A. Abbink, Mark Mulder, and Joost C. F. de Winter. 2015. The effect of haptic support systems on driver performance: A literature survey. IEEE Transactions on Haptics 8:467-479.

Raksincharoensak, Pongsathorn, and Hideo Inoue. 2017. Safety cushion: Context-sensitive hazard anticipation -objectified driving behavior of experienced and careful drivers for developing context-sensing driving assistance systems-. In FAST-zero'17: 4 th International Symposium on Future Active Safety Technology Toward zero traffic accidents, 2017, 20174609.

Rankavat, Shalini, and Geetam Tiwari. 2016. Pedestrians perceptions for utilization of pedestrian facilities - delhi, india. Transportation Research Part F: Traffic Psychology and Behaviour 42:495-499.

Saito, Yucihi, and Pongsathorn Raksincharoensak. 2016a. Shared control in risk predictive braking maneuver for preventing collisions with pedestrians. IEEE Transactions on Intelligent Vehicles 1:314324 . 
Saito, Yucihi, and Pongsathorn Raksincharoensak. 2016b. A shared control in risk predictive braking manoeuvre for preventing collision with pedestrian. In 2016 IEEE International Conference on Systems, Man, and Cybernetics (SMC), 685-690.

Sarter, Nadine B., and David D. Woods. 1995. How in the world did we ever get into that mode? mode error and awareness in supervisory control. Human Factors 37:5-19.

Takae, Yasuhiko, Yoji Seto, Tomohiro Yamamura, Takeshi Sugano, Masahiro Kobayashi, and Kazuto Sato. 2009. Development and evaluation of a distance control assist system with an active accelerator pedal. SAE Int. J. Passeng. Cars - Electron. Electr. Syst. 2:46-55.

Verwey, Willem B., Håkan Alm, John A. Groeger, Wiel H. Janssen, Marja J. Kuiken, Jan Maarten Schraagen, Josef Schumann, Wim van Winsum, and Heinz Wontorra. 1993. Gids functions. In In J. A. Michon (Ed.), Generic Intelligent Driver Support. Taylor \& Francis.

Vlassenroot, Sven, Steven Broekx, Johan De Mol, Luc Int Panis, Tom Brijs, and Geert Wets. 2007. Driving with intelligent speed adaptation: Final results of the belgian isa-trial. Transportation Research Part A: Policy and Practice 41:267-279.

Young, Mark S., Stewart A. Birrell, and Neville A. Stanton. 2011. Safe driving in a green world: A review of driver performance benchmarks and technologies to support 'smart' driving. Applied Ergonomics 42:533-539. 